\title{
Modeling Geographical Anycasting Routing in Vehicular Networks
}

\author{
Alireza Amirshahi ${ }^{1}$, Morteza Romoozi ${ }^{2 *}$, Mohammad Ali Raayatpanah ${ }^{3}$ \\ and Seyyed Amir Asghari ${ }^{4}$ \\ ${ }^{1}$ Department of Computer Engineering, Qom Branch, Islamic Azad University, Qom, Iran \\ [e-mail: Alirezaamirshahi@gmail.com] \\ ${ }^{2}$ Department of Computer Engineering, Kashan Branch, \\ Islamic Azad University, Kashan, Iran \\ [e-mail: mromoozi@gmail.com] \\ ${ }^{3}$ Faculty of Mathematical Sciences and Computer, Kharazmi University, Tehran, Iran \\ [e-mail: raayatpanah@khu.ac.ir] \\ ${ }^{4}$ Computer and Electrical Engineering Department, Kharazmi University of Tehran, Iran. \\ [e-mail: amirasghariel@gmail.com] \\ *Corresponding author: Morteza Romoozi
}

Received August 8, 2019; revised December 13, 2019; accepted January 4, 2020; published April 30, 2020

\begin{abstract}
Vehicular network is one of the most important subjects for researchers in recent years. Anycast routing protocols have many applications in vehicular ad hoc networks. The aim of an anycast protocol is sending packets to at least one of the receivers among candidate receivers. Studies done on anycast protocols over vehicular networks, however, have capability of implementation on some applications; they are partial, and application specific. No need to say that the lack of a comprehensive study, having a strong analytical background, is felt. Mathematical modeling in vehicular networks is difficult because the topology of these networks is dynamic. In this paper, it has been demonstrated that vehicular networks can be modeled based on time-expanded networks. The focus of this article is on geographical anycast. Three different scenarios were proposed including sending geographic anycast packet to exactly-one-destination, to at-least-one-destination, and to K-anycast destination, which can cover important applications of geographical anycast routing protocols. As the proposed model is of MILP type, a decentralized heuristic algorithm was presented. The evaluation process of this study includes the production of numerical results by Branch and Bound algorithm in general algebraic modeling system (GAMS) software and simulation of the proposed protocol in OMNET++ simulator. The comprehension of the result of proposed protocol and model shows that the applicability of this proposed protocol and its reactive conformity with the presented models based on presented metrics.
\end{abstract}

Keywords: Anycast routing, Vehicular networks, Time-expanded networks, Branch and Bound algorithm, Mixed integer programming. 


\section{Introduction}

Vehicular networks are special mobile ad hoc networks (MANET); nodes are vehicles which interact information by creating communication [1]. Vehicular networks have many features including self-organization, self-management, high speed, mobility, and high dynamic topology. Of important applications of these networks, we can refer to safety, traffic management, and assisting the drivers [2, 3, 4]. Routing plays a crucial role in efficiency of VANET networks $[5,6]$. Routing process in vehicular networks is really challenging. Finding the shortest end-to-end route with limited delay and minimum overhead has challenges and limitations. Such challenges are because of high mobility of vehicles, frequency failure of the route, and different obstacles which influence confidence in transferring data and routing [7].

Routing protocols in VANET can be categorized in three groups: multicast, unicast, and anycast. In multicast method the message from one sender are sent to a number of destinations or to a group of interested nodes [8]. In unicast method the purpose is sending data from a source node to a target node $[9,10]$. Anycast method is an important communicative pattern in which the purpose is to send a data packet to at least one of the receivers from among candidate receivers $[11,12]$. The criterion of selecting the best receivers from among receivers is really demanding [10]. One of the most influential criteria in route selection is geographical distance. In geographical routing, each node in the network is aware of its location and those of its neighbors, and of destination or destinations [13]. Therefore, to decide for packet relay, the node selects a neighbor with the shortest distance from destination. In vehicular networks, a large number of studies have been done to find geographical routing in unicast [14] and multicast, but a limited number of studies have been performed on geographical anycast.

Recently, by ever increasing popularity of internet of things (IoT) and development in its communication underlay, a new paradigm for integrating IOT and VANET has been proposed that is called internet of vehicle (IoV) $[8,15]$. Anycasting in IoV can be challenging due to gateway selection in IoV. It means that a vehicle can anycast its sensed data to the potential gateway sets. So, the proposed model and protocol in this paper can be justified for IoV.

For geographical anycast routing to be performed, a problem can be modeled with an indirection graph in a way that vehicles, nodes and physical links among vehicles be links. The weight of each link is the geographic distance of node. The source node selects the nearest destination(s) and direct anycast packet toward them. The high dynamic topology of vehicular networks is probably the most problematic issue for modeling geographical anycast routing in vehicular networks that changes the network over routing $[16,17,18]$.

In order to solve this problem, the life of a topology can be divided into burst regular discrete networks; in every static network, a part of routing process is performed. Therefore, considering the dynamic topology of network, routing can be modeled. In this study, based on mixed-integer linear programming, time-expanded networks have been applied to model the dynamic structure of vehicular networks.

In this article, three different scenarios for geographical anycast can be considered.

1. Exactly-one-destination: in this scenario, each neighboring relay node which has the nearest geographical distance to the nearest destination is selected.

2. At-least-one-destination: In this scenario, all destinations having calculation costs lower than the total average cost of all destinations will be selected and a collection of selected neighbors will be chosen as relay. 
3. K-anycast: in this scenario, only K destination with the lowest cost will be selected and then a collection of the best selected neighbors for $\mathrm{K}$ destination will be computed and the packet will be forwarded to this collection.

For each of the above mentioned scenario, a mixed-integer linear programming, based on time-expanded networks, will be presented.

In order to make use of these models in vehicular networks, a decentralized algorithm must be designed; in this article, for each scenario, a heuristic algorithm has been presented which selects the best neighbor(s) to forward anycast packets to the considered destination(s).

To evaluate the proposed model and related algorithm, the model is first solved by (GAMS) software. The required graph is extracted from simulator to solve the model to compare the results with simulation of algorithm. Finally, the proposed algorithms for these models are simulated by OMNET++ and the results are compared with the results of solving model by GAMS software and are validated. Also, the results are compared to geographic K-anycast destination routing (GKAR) [13], which has been simulated by OMNET++ and compared to the proposed K-anycast destination.

This simulation results of the proposed algorithm nearly support the proposed model. In the other words, the trend of the diagrams related to the model and that of proposed algorithm follow a similar pattern.

The rest the of paper is organized as follows. Section 2 reviews the related work in geographic routing. In sections 3 , the problem description and the suggested model are provided for anycasting over vehicular networks for the three considered scenarios. The proposed algorithm is illustrated in section 4 . The model validation and performance metrics of proposed models presented in sections 5 and 6 , respectively. The results and general evaluation are presented in sections 7 and 8, respectively. Finally, section 9 concludes our work.

\section{Related work}

Anycasting in VANET can be categorized in three mentioned scenarios. In this section, we review previous studies in the VANET based on these scenarios.

\subsection{Anycasting in the VANETs based on exactly-one-destination scenario}

In [19] an Anycasting-Driven Reservation System for electrical vehicles in an urban environment has been proposed. In this system, the goal is finding the best charging station with minimum congestion. It proposes exactly one destination among potential charging station destinations. In [20] a single-copy anycast routing strategy has been proposed. In this protocol, a relay node is selected by higher anycast social distance metric (ASDM). It is a social metric that can balance efficiency and robustness between potential routes. The final routes lead to one of the destinations. So, it can belong to exactly-one anycast scenarios.

\subsection{Anycasting in the VANETs based on at-least-one destination scenario}

In [21], adaptive anycasting solution was proposed for vehicular environments. Geographical and topological information were combined in order to adapt its behavior in network conditions. Regarding V2I connectivity, the sensed information is sent, by vehicles, as individual and independent message to at least one of RSUs. This protocol attempts to select the best RSU. A routing protocol was presented by Gao et al. in [22] for K-anycast communication in wireless sensor networks. To instruct the route detection with 3 outlines for 
$\mathrm{K}$-anycast, they took into account multiple-metrics where a packet is transferred to precisely $\mathrm{k}$ sinks, at most k sinks and at least k sinks. In [23], a parking system has been proposed. The challenge in this system is sending reserve request to at least one of the parking servers. In this at-least-destination anycast scenario, each vehicle broadcasts his request packet and each neighboring receiver sends it to one of the parking servers. In [24] a typical anycast protocol has been proposed over delay tolerant networks (DTN). In this protocol, the goal is sending the packet to at least one of destinations. Each node investigates its 2-hop neighbor for forwarding the packet to the nearest receivers. Distance metric in this protocol is geographical distance. If a node cannot find a better neighbor than itself, it will carry the packet until it finds such a neighbor. In [25] a time constrained anycast protocol has been proposed over DTN. In this protocol, relay nodes use previous and current contacts to decrease transmission costs. In selecting relay node, a combination of delivering probability for one and two hops is considered. This protocol can be categorized in at-least-one destination scenario.

\subsection{Anycasting in the VANETs based on K-anycast scenario}

In [13], a geographic K-anycast routing in WSNs is proposed. They present a geographical routing protocol according to k-anycast communication. The proposed algorithm not only selects the next hop but also, determines the number of destinations. In [22], a routing protocol in WSN for K-anycast communication is proposed. Authors, in this paper, based on the anycast tree scheme, present K-anycast scenario. In [26], authors have focused on minimizing delay in data acquisition over IoV. So, to request the required data chunks, a K-anycast protocol has been proposed. A K-anycast-destination request packet has been obtained from different data chunks, which are sent to the originator simultaneously. Authors in [27] propose a K-anycast protocol over DTN. The proposed protocol considering real-time network topology and the type of request packet selects a route to $\mathrm{k}$ destinations.

\section{Problem description}

In this article, the dynamic structure of vehicular networks has been modeled. Then, based on this model, geographical anycast is modeled by an integer linear programming model. The main applied notations are illustrated in Table 1.

Table 1. NOTATIONS AND DESCRIPTIONS

\begin{tabular}{|c|c|c|c|}
\hline Notations & Descriptions & Notations & Descriptions \\
\hline$x_{i, j}^{t} \in\{0,1\}$ & $\begin{array}{l}x_{i, j}^{t}=1 \text { if the edge lies on path else } \\
x_{i, j}^{t}=0\end{array}$ & $N$ & $\begin{array}{l}\text { Set of vehicles and set of RSUs: } \\
N \in\{V \cup R\}\end{array}$ \\
\hline$\frac{c_{i, j}^{t}}{}$ & The geographic cost of link $(i, j)$ & $T$ & The integer time horizon \\
\hline$O^{1}$ & Source node in the first snapshot & $\delta$ & Set of receiver's \\
\hline$D$ & A set of destinations. & $k$ & Set of different destinations \\
\hline$L Q_{i, j}^{t}$ & Quality of link $(i, j)$ in snapshot $t$ & $H$ & A set of selected neighbors \\
\hline$L D_{i, j}^{t}$ & $\begin{array}{l}\text { Failure probability of link }(i, j) \text { in } \\
\text { snapshot } t\end{array}$ & $c_{(i . j)}$ & Cost of geographic \\
\hline $\operatorname{cap}_{i, j}^{t}$ & Capacity of link $(i, j)$ in snapshot $t$ & $\delta_{(i, i)}$ & Reduction ratio of geographic cost \\
\hline$f_{i}^{t}$ & $\begin{array}{l}f \text { represents the number } \\
\text { transferred packets of node } i \text { in } \\
\text { snapshot } \quad t \quad \\
\text { at-least-one-destination scenario }\end{array}$ & $W_{j}$ & The j-th neighbor of node $O^{1}$ \\
\hline$i d_{i}$ & Identifier of vehicle $i$ & $M$ & The number of neighbors \\
\hline$s_{i}$ & Speed of vehicle $i$ & $\mathrm{D}$ & The number of destinations \\
\hline
\end{tabular}




\begin{tabular}{|l|l||l|l|}
\hline$d_{i}$ & Direction of vehicle $i$ & $\mathrm{H}$ & A set of selected neighbors \\
\hline$m_{k}$ & $\begin{array}{l}\text { The geographical map's link moving } \\
\text { along it in vehicle. }\end{array}$ & $\mathrm{R}$ & Transmission range \\
\hline$v$ & Set of vehicles & $y_{i, j} \in\{0,1)$ & $\begin{array}{l}y_{i, j}=1 \text { if } w j \text { is selected as the next } \\
\text { hop to reach destination } d \mathrm{i}, \\
\text { otherwise } y_{i, j}=0\end{array}$ \\
\hline$R$ & Set of RSUs & demand & Packet size \\
\hline$A$ & Set of links & FPP & failure probability of a path \\
\hline
\end{tabular}

\subsection{Network model and problem definition}

In [28], Amirshahi et al. have solved the problem based on hop count in non-geographical states. In this paper, the focus is on geographical state. A directed graph, $G=(N, A)$, represents vehicular network, where $N \in\{v \cup R\}$, V denotes for the set of vehicles, R shows the set of RSUs and A is the set of links. Each link $(i, j)$ is related to $\left\{\operatorname{cap}_{i, j}, c_{i, j}, L D_{i, j}, L Q_{i, j}\right\}$; $\operatorname{cap}_{i, j}$ shows the link capacity. $c_{i, j}$ show the geographical cost of link (the same as geographic distance), $L D_{i, j}$ is the link failure probability, and $L Q_{i, j}$ refer to link quality between nodes i and $\mathrm{j}$. Each node i, if $i \in N$, could be defined by a set with four members as $\left\{i d_{i}, s_{i}, d_{i}, m_{k}\right\}$; $i d_{i}$ denotes the vehicle identifier, $s_{i}$ illustrates vehicles speed, $d_{i}$ shows its direction, and $m_{k}$ defines the link of the geographical map which moves along it. $i \in R, s_{i}=d_{i}=0$, because RSUs are statistically used at known positions. Regarding the vehicular networks dynamic topology, modeling is through static network is not possible. To tackle this change, we apply the concept of time-expanded networks that contain one copy of the original network for each discrete time step. In this case, we can solve the problem of determining an optimal solution over time in a static network [29]. Therefore, a time horizon $\mathrm{T}$ is required to cover network topology variations. We can modify the suggested model of network as $G^{T}=\left(N^{T}, A^{T}\right)$, in which, $\mathrm{T}$ shows the integer time horizon; this is required to determine the links and nodes regarding parameter of time. $N^{T}$ shows the set of the nodes and $A^{T}$ defines the set of the links over time as:

$(1) \mathrm{N}^{\mathrm{T}}=\mathrm{U}_{\mathrm{t}=1}^{\mathrm{T}-1}\left\{\mathrm{i}^{\mathrm{t}} \mid \mathrm{i} \in V \cup R, \mathrm{t}=1,2, \ldots, \mathrm{T}-1\right\}, \mathrm{A}^{\mathrm{T}}=\mathrm{U}_{\mathrm{t}=1}^{\mathrm{T}-1} A^{t}$, where $A^{t}=\left\{\left(i^{t}, i^{t+1}\right) \mid i \in V, t=1,2, \ldots, T-1\right\} \cup\left\{\left(i^{t}, j^{t}\right) \mid(i, j) \in A, t=1,2, \ldots, T-1\right\}$

It is proposed that network time horizon is classified into various regular static networks. So, in this article, to model the vehicular networks dynamic structure, time-expanding network, [30] is applied. The network time horizon is divided into interconnected slices in these time-expanded networks. With the time period $T \in \mathbb{Z}^{+}$, it can be $=0,1,2 \ldots, T-1$, while each t shows the network's snapshot that is constant. Consider that each snapshot is connected to the next snapshot by corresponding node. For instance, take into account a vehicular 6-node network as illustrated in Fig. 1 and Fig. 2 shows a time-expanding network in 3 snapshots, in which each snapshot shows a different topology. In snapshot $t$, the definition of node $i$ is $\left\{i d_{i}, s_{i}^{t}, d_{i}^{t}, m_{k}\right\}$, each link as $\left\{c a p t_{i, j}, c_{i, j}^{t}, L D_{i, j}^{t}, \mathrm{LQ} Q_{i, j}^{t}\right\}$, while index t is snapshot number.

The geographical cost of link, capacity, quality, and failure probability of link $\left(i^{t}, j^{t}\right)$ for $i \in N, t=1,2, \ldots, T-1$ are denoted by $c_{i, j}^{t}$, $c a p t_{i, j}, L Q_{i, j}^{t}$, and $L D_{i, j}^{t}$, respectively. These criteria for link $\left(i^{t}, j^{t}\right), i \in N, t=1,2, \ldots, T-1$ are represented by $0, \infty, \infty$, and $\infty$, respectively. 
Definition 1: A route from node $i_{0}$ to $i_{p}$ is a order of links $p=$ $\left\{\left(i_{0}, i_{1}\right),\left(i_{1}, i_{2}\right), \ldots,\left(i_{p-1}, i_{p}\right)\right\}$, while each link initial node is similar to the preceding link's terminal node in the sequence, and $i_{0}, \ldots, i_{p}$ are all distinct nodes. Therefore, in the path, each link is conducted toward $i_{p}$ and far from $i_{0}$.

Definition 2: considering definition 1, anycast route is a route in which a packet is sent to at least one of destinations and the route is the best possible one.

Definition 3: When the path links probability is not higher than the threshold defined by $L D$, the route is named feasible regarding failure probability. Because links are supposed to be independent, we define a path failure probability as:

$$
F P P=\prod_{t=\mathbf{0}}^{T-1} \prod_{(i, j) \in A}\left(L D_{i, j}^{t}\right)^{x_{i, j}^{t}}+\prod_{t=\mathbf{0}}^{T-1} \prod_{i \in N}\left(L D_{i, i}^{t}\right)^{x_{i, i}^{t}}
$$

Each link $(i, j)$ is related to a decision variable $x_{i, j}^{t}$ when $x_{i, j}^{t}$ defines 1 when the link lies on the path and zero on the other hand. As observed in Fig. 2, path $p_{1}: 1^{1} \rightarrow 2^{1} \rightarrow 2^{2} \rightarrow 4^{2} \rightarrow$ $4^{3} \rightarrow 6^{3}$ fails having the probability of $0.0225(0.15 * 0.25 * 0.6=0.0225)$; and path $\mathrm{p}_{2}: 1^{1} \rightarrow$ $3^{1} \rightarrow 3^{2} \rightarrow 5^{2} \rightarrow 5^{3} \rightarrow 6^{3}$ fails having the probability of $0.009(0.15 * 0.2 * 0.3=0.009)$. If the minimum failure probability constant is considered as equal to $\mathrm{LD}=0.01$, so $p_{1}$ is not considered feasible while path $p_{2}$ is regarded feasible. Therefore, a path is regarded feasible if:

$$
\prod_{t=\mathbf{0}}^{T-1} \prod_{(i, j) \in A}\left(L D_{i j}^{t}\right)^{x_{i j}^{t}}+\prod_{t=\mathbf{0}}^{T-1} \prod_{i \in N}\left(L D_{i i}^{t}\right)^{x_{i i}^{t}} \leq L D
$$

Equation 3, is a nonlinear relation that can be converted to a linear relation via algorithm techniques:

$$
\log \left(\prod_{t=\mathbf{0}}^{T-1} \prod_{(i, j) \in A^{t}}\left(L D_{i j}^{t}\right)^{x_{i j}^{t}}+\prod_{t=\mathbf{0}}^{T-1} \prod_{i \in N}\left(L D_{i i}^{t}\right)^{x_{i i}^{t}}\right) \leq \log (L D)
$$

Hence, we have:

$$
\sum_{t=\mathbf{0}}^{T-1} \sum_{(i, j) \in A^{t}} x_{i j}^{t} \log \left(L D_{i j}^{t}\right)+\sum_{t=\mathbf{0}}^{T-1} \sum_{i \in N} x_{i i}^{t} \log \left(L D_{i i}^{t}\right) \leq \log (L D)
$$

\subsection{Modeling anycast problem}

In geographical anyacst modeling, considering each scenario, the purpose is to minimize the cost to find the best path.

\subsubsection{Modeling anycasting in exactly-one-destination scenario}

In this scenario, it is aimed to forward the packet to the greatest destination among destinations group. Finding the appropriated destination may be performed based on such factors as geographic cost, link duration, link quality, etc. As illustrated in Fig. 3, the problem can be minimized to a single destination, which is the shortest path, by addition of a dummy destination and the link of other destinations having zero cost. Therefore, the best destination can be selected out of a set of candidate destinations $\left(d_{1}, d_{2}, d_{3}\right)$. To make the modeling simple, the cost function just includes link cost and other objectives are regarded in the constraint. So we can claim that this is a single objective model. 


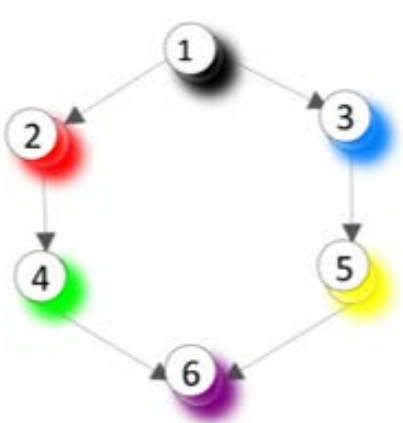

Fig. 1. A vehicular network with source 1 to destination 6

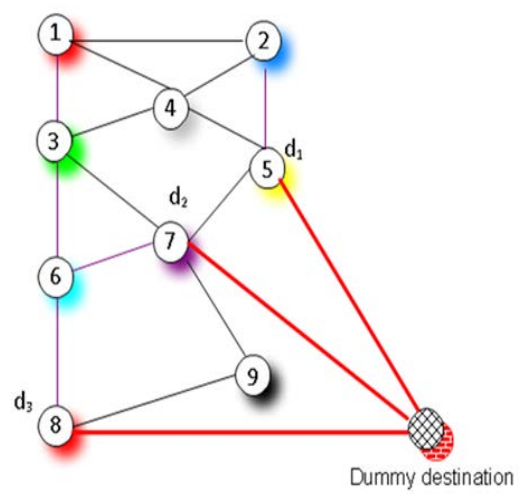

Fig. 3. Packet broadcasting to the best destination among all other destinations

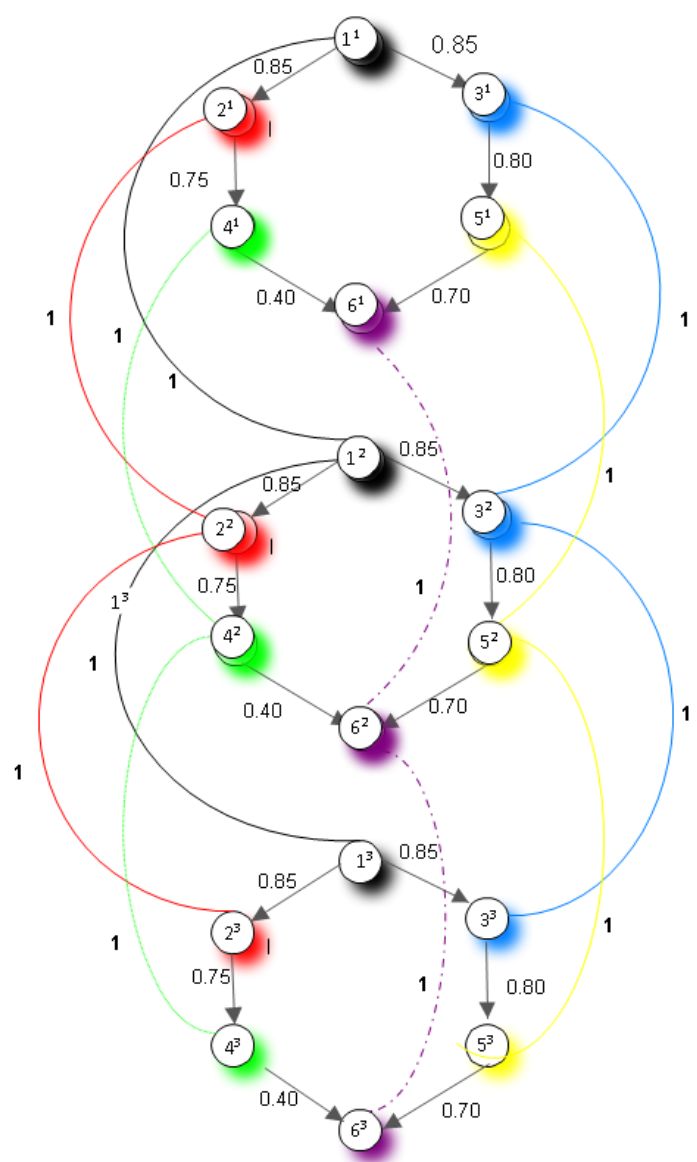

Fig. 2. Time-expanded network of Fig. 1

This scenario is defined as an integer linear programming as described below:

$$
\begin{gathered}
\min \sum_{t} \sum_{(i, j) \in A} c_{i, j}^{t} x_{i, j}^{t} \\
\sum_{\left\{j \mid(\mathrm{i}, j) \in A^{t}\right\}} x_{i, j}^{t}-\sum_{\left\{j \mid(j, i) \in A^{t}\right\}} x_{j, i}^{t}=\left\{\begin{array}{ll}
1, & i=O^{1}, \\
-1, & i=D \\
0, & 0 . W,
\end{array} \quad \forall t=1,2, \ldots T-1,\right. \\
\sum_{t=1}^{T-1} \sum_{(i, j) \in A^{t}} x_{i j}^{t} \log \left(L D_{i j}^{t}\right)+\sum_{t=1}^{T-1} \sum_{i \in N} x_{i i}^{t} \log \left(L D_{i i}^{t}\right) \leq \log (L D), \\
L Q_{i, j}^{t} * x_{i, j}^{t} \geq L Q, \quad \forall(i, j) \in A^{t}, \quad t=1,2, \ldots T-1, \\
\operatorname{demand} * x_{i, j}^{t} \leq \operatorname{cap} p_{i, j}^{t}, t=1,2, \ldots T-1, \\
\sum_{t=1}^{T-1} x_{i^{t}, i^{t+1}=1, \forall i \in V,}
\end{gathered}
$$




$$
\begin{gathered}
x_{i, j}^{t} \in\{0,1\}, \quad \forall(i, j) \in A^{t}, t=1,2, \ldots T-1, \\
x_{i^{t}, t^{t+1}} \in\{0,1\}, \quad i \in N, t=1,2, \ldots T-1,
\end{gathered}
$$

The cost of path to dummy destination is minimized by cost function equation 6 . The packets transmit to destination is guaranteed by constraint 7 as a flow constraint, while, in the first snapshot, $o^{1}$ is the source node. In this constraint, it is possible to use the discrete periods to make various levels of services quality. For example, the packets reaching the destination in $[t, t+\delta]$ causes to the quality lying in level A. As it can be observed in constraint 8 , the links with a greater link quality than threshold LD's are permitted to be applied. Like constraint 8, in constraint 9, we consider minimum link quality. In equation 10, it is guaranteed that sum of packets existing on a link never exceeds the capacity in total. In constraints 11, it is not possible to use the link between node i to itself in the subsequent snapshot over 1 time. Logical restrictions on $x_{i, j}^{t}$ and $x_{i^{t}, i}{ }^{t+1}$ variables are presented in constraint 12 and 13 respectively.

\subsubsection{Modeling anycasting in at-least-one-destination scenario}

In this scenario, the packet is transferred to a subset of a collection of destinations D. By this scenario, the probability of packet delivery to at least one of the destinations is increased.

In Fig. 4 , when $\mathrm{b}$ is anycast packet forwarded, $d_{1}$ and $d_{2}$ are destinations, a copy of packet will be transferred to c, because, from among all destinations it is the best destination. Another one will be sent to e, because of having a lower cost path to $d_{2}$ compare to $\mathrm{f}$. If, in c, the packet is lost, it can get to $d_{2}$ via e. Each forwarder node in this scenario can transfer its packet to over 1 neighbor. Considering that each forwarder like i can send its packet to $f_{i}^{t}$ neighbors in snapshot $\mathrm{t}, f_{i}^{t}$ can be different for each $\mathrm{i}$ and $\mathrm{t}$. Note that $f_{i}^{t}$ can be equal to the minimum cut capacity. However, optimizing $f_{i}^{t}$ requires further study. As its value is regarded constant and pre-defined in this condition.

We can formulate the scenario as an integer linear programming:

$$
\min \sum_{t} \sum_{(i, j) \in A} c_{i, j}^{t} x_{i, j}^{t}
$$

S.t.

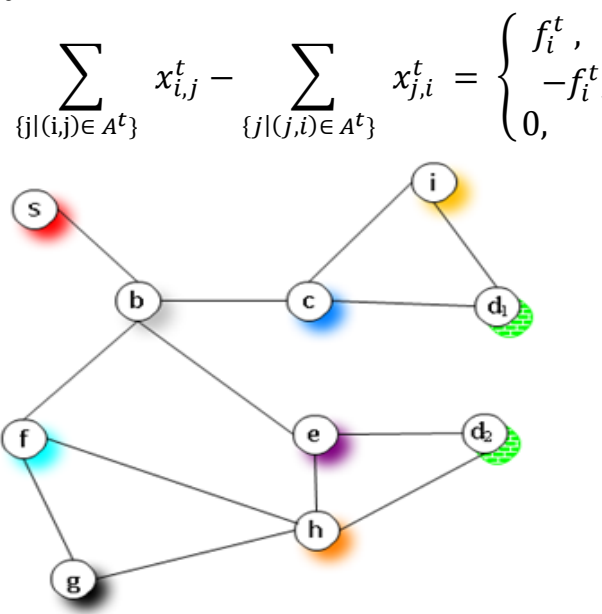

Fig. 4. Sending the packet to a subset of destinations D.

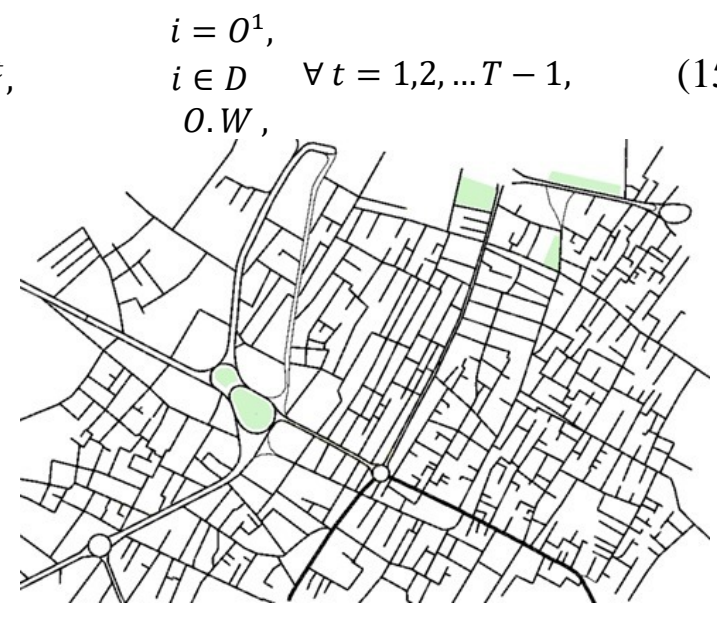

Fig. 5. city map in SUMO. 
In equation14, cost function is the same as cost function of previous model. Constrain 15 is like the constraint in the previous scenario, however, each node like $i$ is able to transfer $f_{i}^{t}$ packets to the related neighbors. Other constraints is the same roles as stated in the previous scenario.

\subsubsection{Modeling anycasting K-anycast destination scenario}

The purpose here is to send message to at least $\mathrm{K}$ destination of $\mathrm{n}$ possible destinations $(\mathrm{k}<\mathrm{n})$, while a dummy node is regarded and all destinations are connected to it by capacity 1 and cost 0 . The input capacity is regarded as $\mathrm{K}$. Thus, the provided model is adjusted as:

This scenario is expressed as an integer linear programming as:

S.t.

$$
\min \sum_{t} \sum_{(i, j) \in A} c_{i, j}^{t} x_{i, j}^{t}
$$

$$
\begin{aligned}
& \sum_{\left\{j \mid(i, j) \in A^{t}\right\}} x_{i, j}^{t}-\sum_{\substack{\left\{\mid(j, i) \in A^{t}\right\} \\
=1,2, \ldots T-1,}} x_{j, i}^{t}=\left\{\begin{array}{cc}
k, & \begin{array}{c}
i=O^{1}, \\
-k,
\end{array} \quad i=D, \quad \begin{array}{l}
t \in D, \\
O . W
\end{array}, \quad \forall t
\end{array}\right. \\
& x_{i, D}^{T} \leq k, \quad \forall i \in \delta,
\end{aligned}
$$

Constraint 17 shows that the dummy destination node needs $\mathrm{k}$ flows, constraint 18 defined the capacity of input links to dummy to $1, \delta$ shows the set of receivers while $\mathrm{K}$ various destinations are applied. Other constraints have the similar roles as expressed in the former scenario.

\subsection{Time complexity of the models}

All the 3 suggested models and their binary variables are contained in the mixed integer programming branch. Some floating points and some integer variables exist in these models. They are aimed to find maximum or minimum of a linear cost function over a solution space with linear constraints. Nevertheless, the space is not convex due to the integer discrete variables.

\section{Proposed algorithm}

To present a decentralized algorithm that is required by such network, a node should decide in each snapshot independent of the other snapshot. So in this selection, without condisordering balance constraint 7, a decentralized algorithm is designed. In this algorithm, the main problem is selecting the next neighbor(s) to forward anycast packet to the destination. This selection (considering the omission of constrain 7) is done with just considering local information of each node and geographic address of destinations without regarding the next snapshots.

The proposed algorithm was inspired from [13]. Then the proposed algorithm was customized to be adapted to our model. At first, all destinations are considered as a set of target destinations. Then in turn, frequently from the farthest destination, this set reduces until the cost decreases. To calculate the geographical cost of a set of candidate destination, all neighbors are created to be selected as relays. This set also reduces from the farthest neighbor until the total cost of the set decreases. The manner of the cost calculation will be explained in 
details in the next section. This algorithm can cover three proposed scenarios in section 3.

\subsection{Problem formulation}

In order to present a decentralized algorithm, we are required to make some changes in the proposed model. In other words, each node should select the best neighbor(s) independently; the goal function for the presented algorithm is illustrated in equation 19. To put it simply, the purpose is to find the lowest cost route and to meet constraints as link quality and link duration.

$$
\min \sum_{i=1}^{|D|} \sum_{j=1}^{m} y_{i, j}\left(\delta_{i, j} * Q(H)\right)
$$

- $y_{i, j} \in\{0,1\}$, so if $w_{j}$ is selected as the next step for destination i, $y_{i, j}=1$ otherwise $y_{i, j}=0$

- $\delta_{i, j}$ is the maximum ratio of geographic cost reduction, from source node $O^{1}$ with selecting $\mathrm{w}_{\mathrm{j}}$ as the next step toward destination $d_{i}$, is obtained from equation 20:

$$
\delta_{i, j}=\frac{c\left(O^{1}, d_{i}\right)-c\left(w_{j}, d_{i}\right)}{c\left(O^{1}, d_{i}\right)}
$$

- $\mathrm{C}\left(0^{1}, d_{1}\right)$ geographic cost is between source node $O^{1}$ and destination $d_{1}$

From the proposed algorithm, the related parameters and other required variables are defined as follows:

The total number of destination(s) that can be allocated with the selection of next step is illustrated in equation 21:

$$
\sum_{i=1}^{D} \sum_{j=1}^{m} y_{i, j}
$$

Therefore, reduction ratio of geographic cost is obtained from equation 22 in which $\mathrm{m}$ is the number of neighbors and $\mathrm{D}$ is the number of destinations.

$$
\delta=\sum_{i=1}^{\mathrm{D}} \sum_{j=1}^{m} y_{i, j} \delta_{i, j}
$$

Equation 23 shows link failure probability between source node and each of the selected neighbors. In case there are a number of neighbors, the neighbor having the highest link failure probability is selected. $\mathrm{H}$ is the number of selected neighbors.

$$
\mathrm{LD}(H)=\underbrace{\max }_{w_{j} \in H} l d\left(O^{1} \cdot w_{j}\right)
$$

In [31], it has been showed that $L D_{i j}$ value is a function of distance. Similarly, link quality $(\mathrm{LQ}(H))$ can be illustrated as a function of distance. Equation 24, shows that if there are a number of neighbors, the neighbor having the highest link quality is selected.

$$
\mathrm{LQ}(H)=\underbrace{\max }_{w_{j} \epsilon H} l q\left(O^{1} \cdot w_{j}\right)
$$

In equation 23 and 24، it is defined that if there are a number of routes, the route having the highest $L Q$ and $L D$ is selected. Equation 25 shows that $Q(H)$ is neighbor selection quality which is a function of $L Q$ and $L D$. This function can be considered as weighted sum or etc. In order to keep generality, it is defined in equation 25 . 


$$
\mathrm{Q}(H)=\mathrm{F}(\mathrm{LD}(\mathrm{H}), \mathrm{LQ}(\mathrm{H}))
$$

\subsection{Algorithm of proposed protocol}

The proposed algorithm has been illustrated by a flowchart in Fig. 6. In the initial phase, first all destinations belong to set $\mathrm{D}$ and all neighbors to set $\mathrm{N}$. Variable $\mathrm{B}$ is overall cost and $\mathrm{C}$ is intermediate cost for selected neighbor sets. Then, the cost for neighbor set $\mathrm{N}$ and destination set $\mathrm{D}$ is calculated. Equation 22 describes how this cost can be calculated. Then the farthest neighbor is removed until the cost $\mathrm{C}$ is decreased. If, by decreasing neighbor set $\mathrm{N}$, the cost dose not decrease any more, the destination set $\mathrm{S}$ will decrease by removing the farthest destination until the overall cost B decreases.

There are three terminating nodes in the flowchart. The first one, when the number of remaining destinations in $\mathrm{D}$ is equal to $\mathrm{k}$ and the selected scenario is k-anycast. The second one, when, by decreasing $\mathrm{D}$, the overall cost does not decrease and the selected scenario is at-least-one. Finally, the third one, when there is only one destination in $\mathrm{D}$ and, therefore the algorithm is terminated by exactly-one scenario.

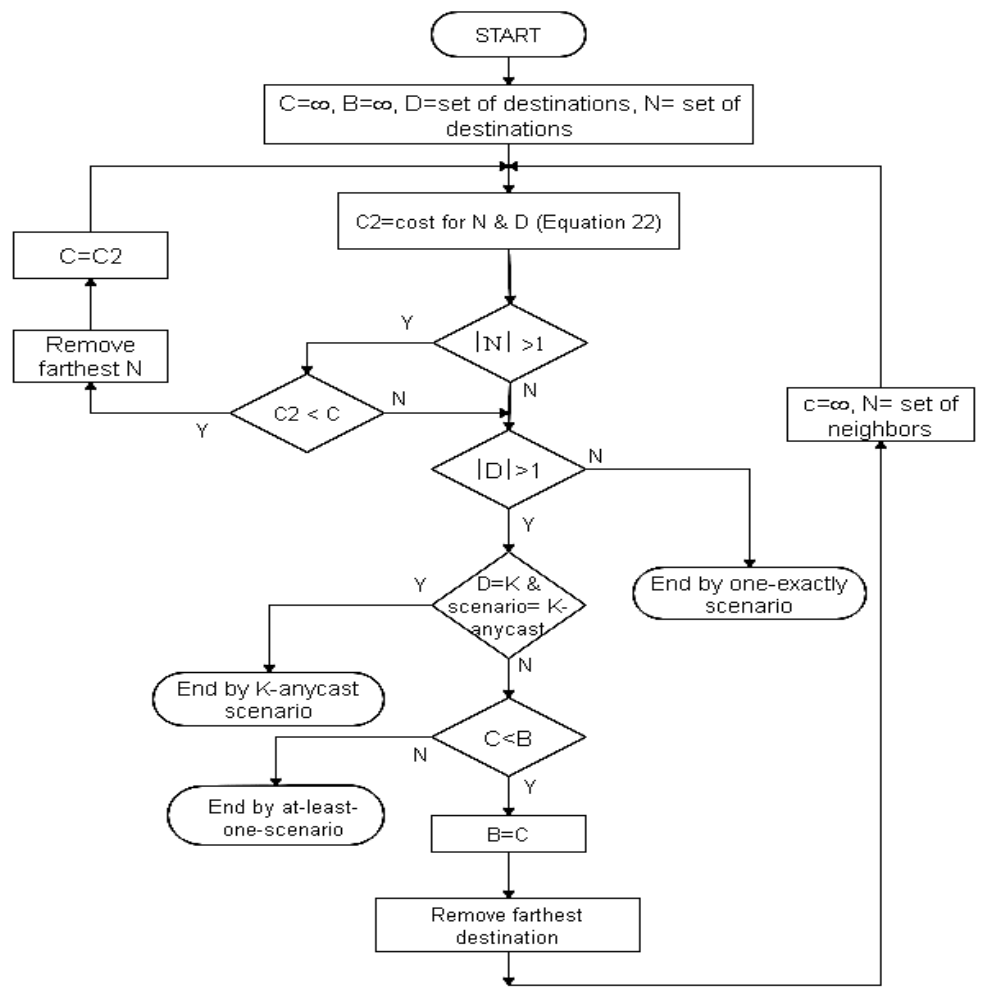

Fig. 6. Flowchart of the algorithm of selecting scenario(s)

\subsubsection{Time complexity of the proposed algorithm}

As it can be observed in flowchart diagram in Fig. 6, the equation 22 should be claculated $\mathrm{m} * \mathrm{D}$ times in worth case. For its calculation, $\mathrm{m} * \mathrm{D}$ is needed too. So, if the graph is complete, we will have $\mathrm{O}\left(n^{2} D^{2}\right)$ in worth case. The number of neighbors and destinations are denoted by $\mathrm{m}$ and $\mathrm{D}$, respectively. 


\section{Model validation and algorithm analysis}

To validate the proposed models and algorithm, the evaluation process in this article is two folds. Firstly, the proposed models are solved by Branch and Bound algorithm in GAMS software and numerical results produced.

Branch and Bound is regarded as a systemic technique to solve integer programming problems. Branch and Bound a divide and conquer attitude are applied to find a set of suitable integer solutions.It should be noted that it applies bounds on the optimal cost, instead of finding the entire suitable set, to refrain from exploring specific parts of the set of suitable integer solutions. Secondly, the proposed algorithm is implemented and evaluated by OMNET++ simulator and its simulation results are compared with numerical results to validate the model.

For producing numerical results two graph is generated. The first one, known as random network is randomly created and the cost of each link is half the transmission range between two nodes. OMNET++ simulator produces simulated network (second network) to approach realistic situation. This simulator is linked to SUMO to create vehicle movement in a real map. The cost of each link is the geographic distance between two nodes. All nodes and the related coordination are written in a file in every $\mathrm{x}$ second. $x$ is the networks sampling rate. In snapshot $\mathrm{K}$, each node is attached to selfsame, one in snapshot $\mathrm{K}+1$ having zero delay and cost.

To calculate each links delay in both networks, equation 27 is utilized and the cost of each link is determined by equation 28.The input for GAMS software is provided by these extracted networks. This software is applied to solve the mix integer models.

All tests are done in terms of different mobility speed and the number of various nodes. In accelerating vehicle mobility speed, as speed rises, so does sampling rate. The number of snapshots rises too. Fig. 5 depicts a part of the real city map applied in SUMO which type of road includes curve, straight, intersection, and etc. Table 2 depicts simulation parameters.

Table 2. SIMULATION PARAMETERS

\begin{tabular}{|c|c|}
\hline Parameter & Value \\
\hline \hline Network area & $5000 \mathrm{~m} * 5000 \mathrm{~m}$ \\
\hline Number of vehiculars & $50,150,200,250,300$ \\
\hline Transfer rate & $100 \mathrm{~m}$ \\
\hline Time of simulation & $600 \mathrm{~s}$ \\
\hline Number of snapshots & 100 \\
\hline MAC Layer Protocol & IEEE 802.11 \\
\hline Speed & $6,9,12,15,18 \mathrm{~m} / \mathrm{s}$ \\
\hline Data payload & 512 bytes/packet \\
\hline RSU Transmission area & $200 \mathrm{~m}$ \\
\hline Simulaton of traffic & SUMO \\
\hline Simulation of vehicular network & Veins 2 \\
\hline
\end{tabular}

\section{Performance metrics of anycasting}

There exist various metrics to assess anycast protocols. Regarding the proposed models, four metrics are given. 


\subsection{Packet delivery ratio}

The metric can compute the ratio of successful delivery of packets to destinations. This metric can be calculated by equation 26. The sum of packets getting to destinations is divided by packets exiting from the senders.

$$
P D R=\frac{\sum_{\forall i, \forall t \in T, j \in D} x_{i, j}^{t}}{\sum_{\forall j, \forall t \in T, i=s^{1}} x_{i, j}^{t}}
$$

\subsection{Average delivery delay}

The average delay in delivering packets successfully is computed by this metric. It is named end-to-end delay. It can be computed by equation 27 by dividing the delay sum for the whole successfully found paths via counting them.

$$
A D D=\frac{\sum_{\forall e_{i, j}^{t} \in \text { Path }_{a c}} \operatorname{Delay}\left(e_{i, j}^{t}\right)}{\mid \text { path }_{a c} \mid}
$$

\subsection{Average delivery cost}

The metric is defined by equation 28 which is obtained by dividing the total cost of routs by the number of them.

$$
A D C=\frac{\sum_{\forall e_{i, j}^{t} \in \operatorname{Path}_{a c}} \operatorname{cost}\left(e_{i, j}^{t}\right)}{\left|p a t h_{a c}\right|}
$$

\subsection{Average hop count}

The average hop count from the source to the destination is called average path length or hop count. $e_{i, j}^{t}$ is a link in which the packet flows. Equation 29 depicts the number of steps from the source node to destination. The denominator of equation 29 shows the number of routes.

$$
A H C=\frac{\sum_{\forall e_{i, j}^{t} \in \text { Path }_{a c}} e_{i, j}^{t}}{\mid \text { path }}
$$

\subsection{Analyzed scenarios}

Analyzed scenarios include three proposed models by 2 input graphs and a proposed protocol for each model and K-anycast protocol proposed in [13]. They are as follows:

- NRGRN-S1: Numerical results of geographical model by random network in scenario1

- NRGSN-S1: Numerical results of geographical model by simulated network in scenario1

- GAP-S1: Geo-anycast protocol in scenario1

- NRGRN-S2: Numerical results of geographical model by random network in scenario2

- NRGSN-S2: Numerical results of geographical model by simulated network in scenario2

- GAP-S2: Geo-anycast protocol in scenario2

- NRGRN-S3: Numerical results of geographical model by random network in scenario3

- NRGSN-S3: Numerical results of geographical model by simulated network in scenario3

- GAP-S3: Geo-anycast protocol in scenario3

- GKAR-S3: geographic K-anycast destination routing in scenario3 


\section{Results}

\subsection{Average link change}

Topology variation is a key factor influencing the network protocols performance [32, 33]. To determine this metric, first $L C(i, j)$ determined as the average number of times altering the link within the two nodes of $\mathrm{i}$ and $\mathrm{j}$ (broken or connected) is defined. $\mathrm{LC}_{\mathrm{t} / \mathrm{t}-1}(\mathrm{i}, \mathrm{j})$ in equation 30 is equal to 1 in case changing the link between the node $i$ and $\mathrm{j}$ from connected to broken or vice versa in snapshot $t$ in comparison to snapshot $t-1$. For each possible link, equation 31 average the variation. This metric is not associated with an anycast protocol, however, it is able to prove the outcomes of other metrics. In Fig. 7, we have illustrated the metric for simulated and random networks. As the nodes continual movement produces the network, the link variation in random network is greater compared to the simulated network. A novel graph in each snapshot represents the random network and its related structure totally alters.

Based on Fig. 8, by increasing the number of snapshots, more links alter between two nodes. Hence, the average of link change will increment as a result of increasing the nodes speed.

$$
\begin{gathered}
L C(i, j)=\sum_{t=0}^{T} L C_{t / t-1}(i, j) \\
A L C=\frac{\sum_{\forall(i, j) \in V} L C(i, j)}{N(N-1) / 2}
\end{gathered}
$$

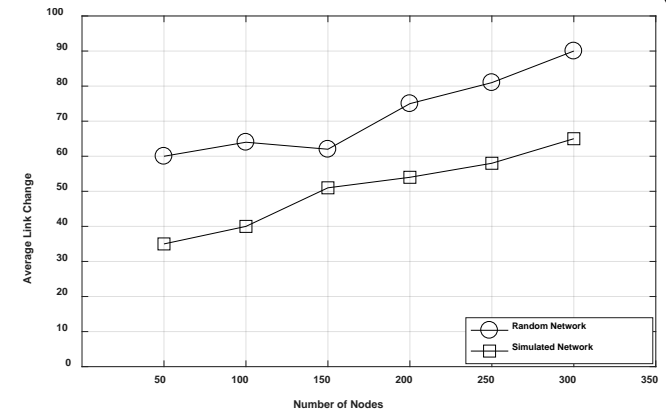

Fig. 7. Average link variations with alteration of the numbers of nodes.

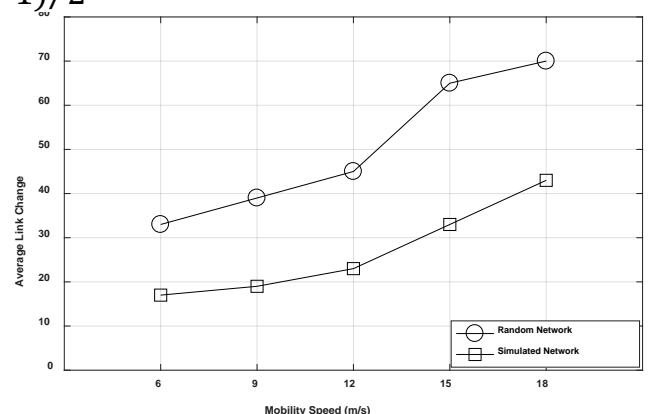

Fig. 8. Average link change with various mobility speed

\subsection{Average hop count}

Equation 29 shows the manner of calculating average hop count. In Fig. 9, 11, and 13, the average hop count, by a various number of nodes, has been illustrateded for each of the proposed scenarios. In NRGRN the nodes are produced by random network. As topology changes and link changes are really high in random network, the average number of hops is more than that of other diagrams. Also, the average hop count in NRGSN and GAP is less than that of NRGRN. In GAP, benefiting the proposed algorithm, the average number of hops has a similar process to the diagrams obtained from GAMS but results are not completely identical. Regarding that this diagram is the simulation of distribution algorithm of geographical model, we expect similar results. There are some efficiency parameters in NRGSN model. That is why the results obtained from GAP \& NRGSN are not totally identical. Amongst the scenarios, $\mathrm{K}$-anycast destination possesses the highest number of hops. This is after an at least one with the last one including exactly-one. Based on calculating the best route for the best destination, exactly-one-destination scenario has minimum hops. Nevertheless, K-anycast destination scenario includes the highest hops as a result of creating paths to k destinations. Fig. 10, 12 
and $\mathbf{1 4}$ depict the average hop count by a various mobility speed of nodes. As the mobility speed rises, so does the process of average number of hops. It is of course expected because of topology fast changes and changes of the route in each hop. Similarly, the number of hops in NRGRN is more than that in the other two diagrams. Also, the variation of the diagrams related to the proposed algorithm and GKAR [13] have a similar pattern.

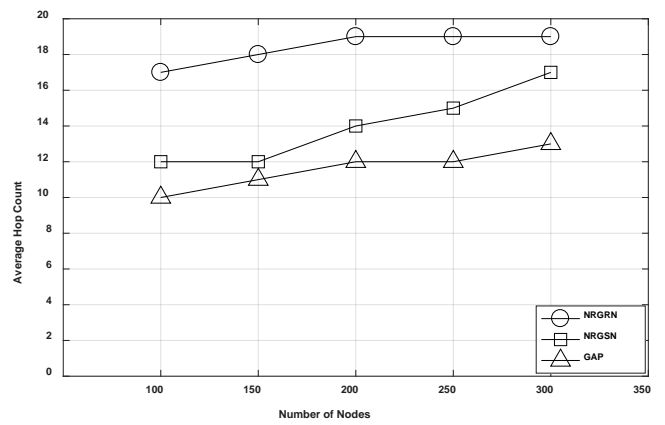

Fig. 9. Average hop count against the numbers of nodes (exactly-one-destination scenario)

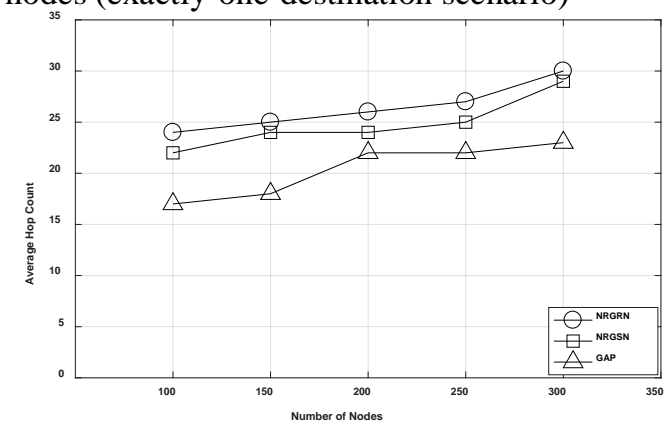

Fig. 11. Average hop count against the numbers of nodes (at-least-one-destination scenario)

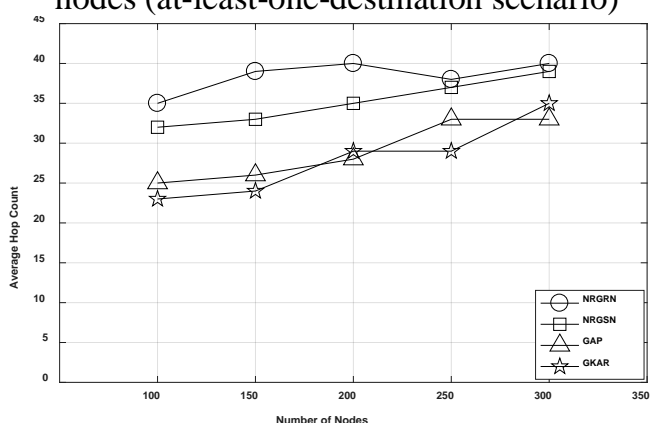

Fig. 13. Average hop count against the numbers of nodes (K-anycast destination scenario)

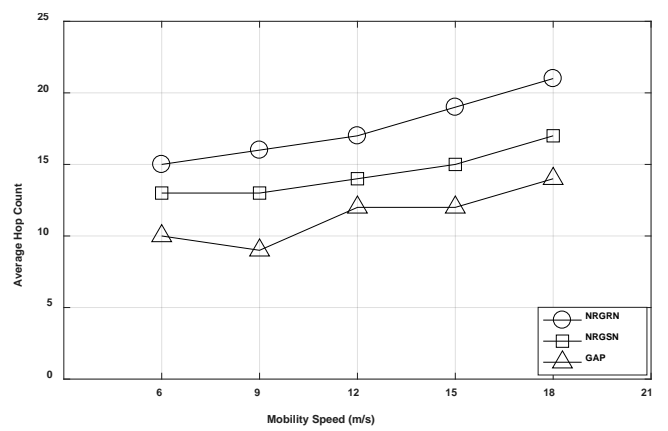

Fig. 10. Average hop count against the mobility

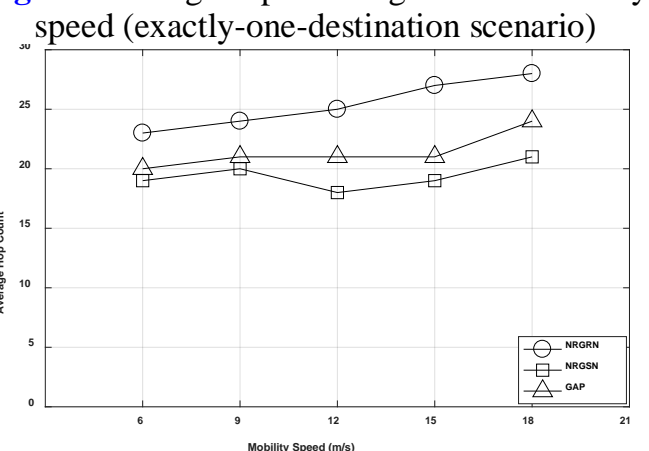

Fig. 12. Average hop count against the mobility speed (at-least-one-destination scenario)

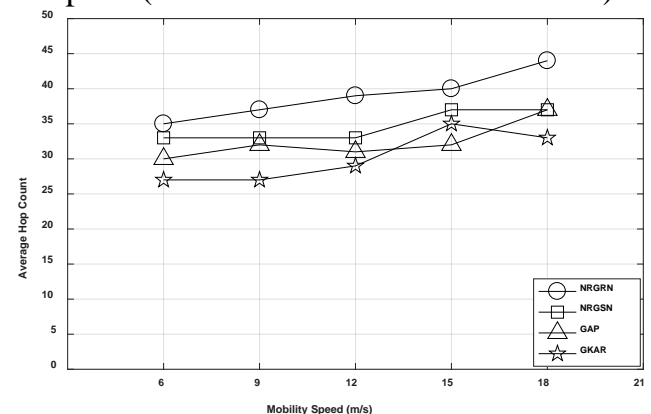

Fig. 14. Average hop count against the mobility speed (K-anycast destination scenario)

\subsection{Average delivery delay}

According to equation 27, the average packet delivery, by a various number of nodes for each of three scenarios is depicted in Fig. 15, 17, and 19. Incrementing the number of nodes, rises the delay in both networks. The average delay of packet delivery in diagrams NRSRN and GAP is less than that of NRGRN; in GAP and NRGSN, a route is selected to deliver the packet that is nearer geographically. So the selected routes will have lower propagation delay. Amongst the scenarios, greater delay of K-anycast destination could be expected. It is caused 
by the fact that the message must be provided to $k$ destinations resulting in the creation of $\mathrm{k}$ routes or a tree with k leaves. Fig. 16, 18 and 20, illustrate that with an increase in mobility speed of nodes, the average delivery delay increases. It is expected considering topology fast changes and changes of the routes in each hop. In this figure similarly, the number of hops in diagram NRGRN is more than that in the other two diagrams. In K-anycast destination scenario, as the packet must cross K-different routes to reach K-destination, the average packet delivery delay is more than the other two scenarios. Two diagrams related to the proposed algorithm and GKAR [13] have a similar pattern.

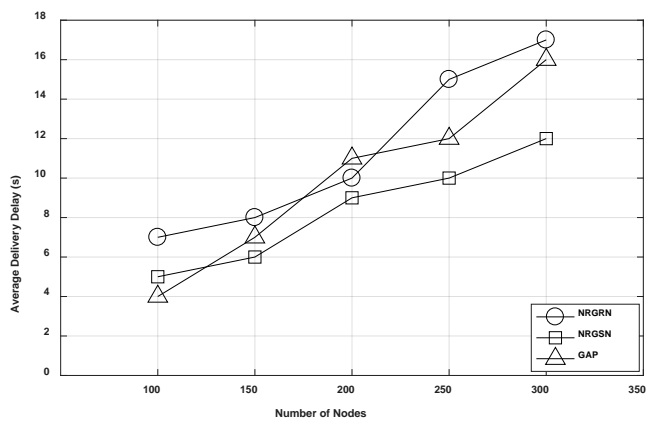

Fig. 15. Average Delivery Delay with different numbers of nodes (exactly-one-destination scenario)

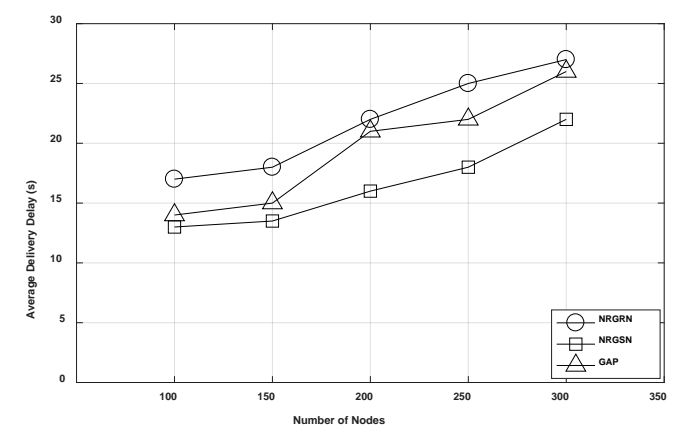

Fig. 17. Average Delivery Delay with different numbers of nodes (at-least-one-destination scenario)

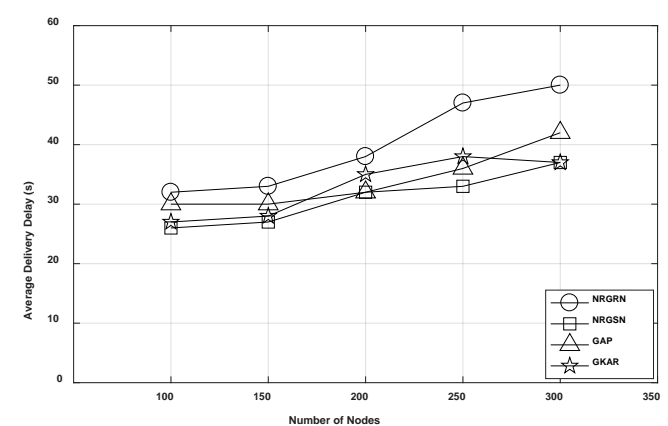

Fig. 19. Average Delivery Delay with different numbers of nodes (K-anycast destination scenario)

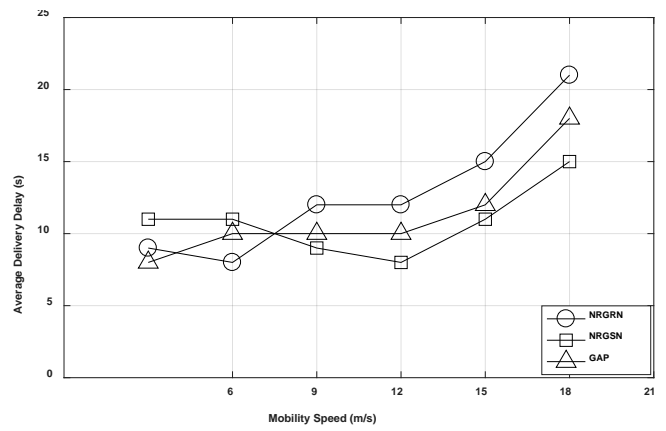

Fig. 16. Average Delivery Delay with different mobility speed (exactly-one-destination scenario)

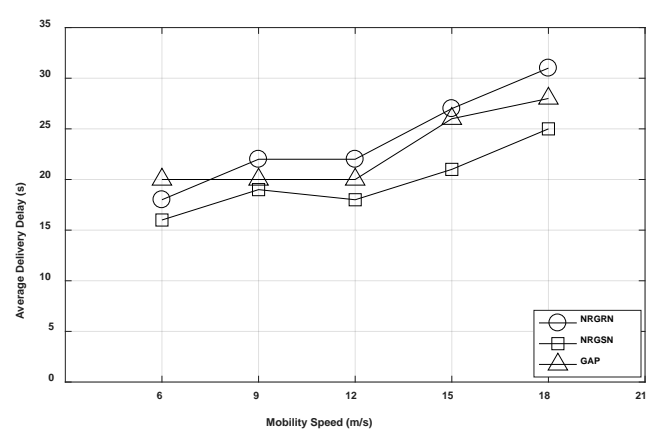

Fig. 18. Average Delivery Delay with different mobility speed (at-least-one-destination scenario)

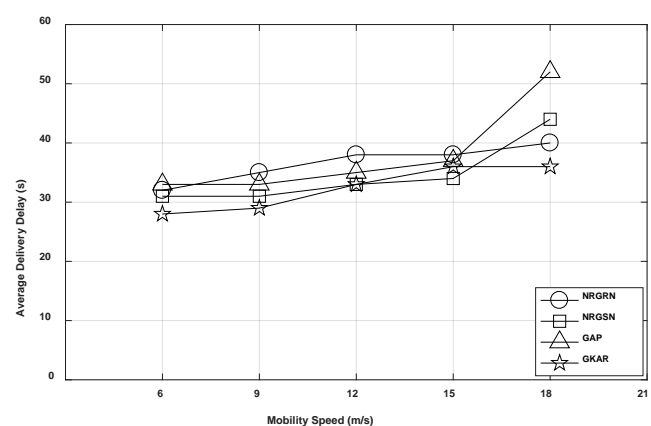

Fig. 20. Average Delivery Delay with different mobility speed (K-anycast destination scenario) 


\subsection{Average delivery cost}

Fig. 21, 23, and 25 show the average packet delivery cost by a various number of nodes in three proposed scenarios. Increasing the number of nodes raises the delivery cost, resulting from the growth in the path length. As a result of topology changes and link changes, the average packet delivery cost in NRGRN is more than that in the other two diagrams. In GAP and NRGSN, a route is selected for packet delivery which is nearer geographically; in these two diagrams, the average packet delivery cost is lower. Because of some effective parameters in simulator, the results obtained from diagrams NRGSN and GAP are not completely identical. Amongst the scenarios, similar to the former metric, K-anycast destination states the highest cost, after at-least-one and exactly-one-destination scenarios. Because our algorithm in scenaro 3 is similar to GKAR [13], they have almost similar variations.

Fig. 22, 24, and 26, illustrate the average packet delivery cost by a various mobility speed of nodes. As node mobility speed increases, the average packet delivery cost falls. Considering equation 29, in NRGRN, because of having a great number of hops compared to the other two diagrams, the average packet delivery cost is higher.

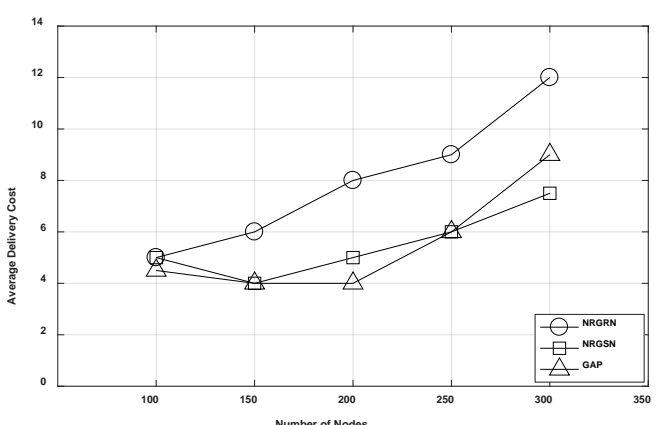

Fig. 21. Average Delivery Cost with different numbers of nodes (exactly-one-destination scenario)

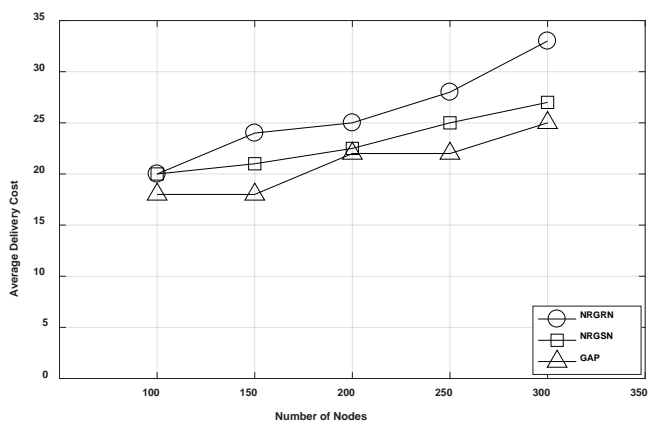

Fig. 23. Average Delivery Cost with different numbers of nodes (at-least-one-destination scenario)

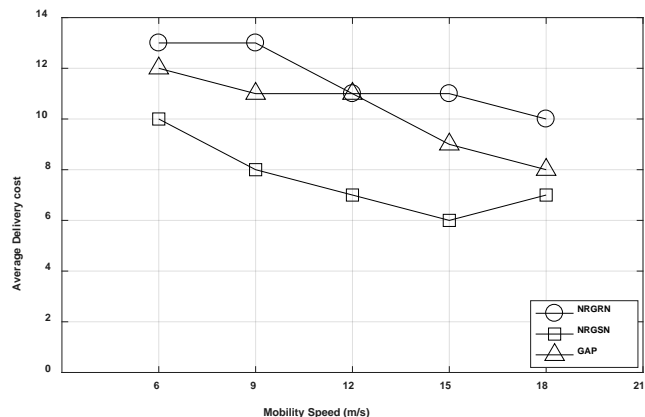

Fig. 22. Average Delivery Cost with various mobility speed (exactly-one-destination scenario)

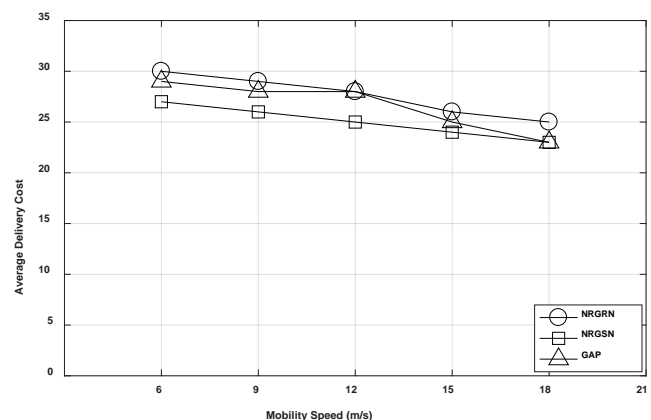

Fig. 24. Average Delivery Cost with different mobility speed (at-least-one-destination scenario) 


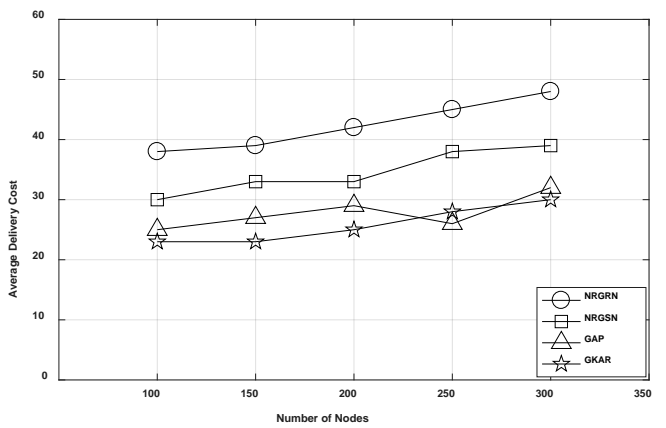

Fig. 25. Average Delivery Cost with different numbers of nodes (K-anycast destination scenario)

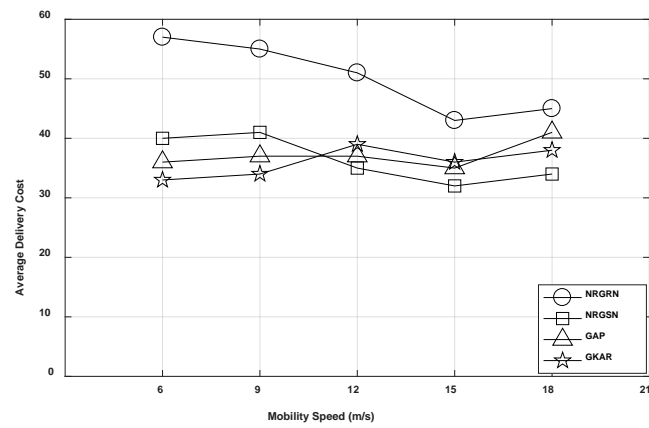

Fig. 26. Average Delivery Cost with different mobility speed (K-anycast destination scenario)

\subsection{Packet delivery ratio}

Fig. 27, 29, and 31 illustrate packet delivery ratio by a various number of nodes. As the number of nodes increases, packet delivery ratio rises too. It is expected regarding the growth in network size and neighborhood. In diagram NRGRN in which a random graph was applied, the packet delivery ratio is greater compared to NRGSN and GAP diagrams in which a simulation graph was applied. In random graph, link and topology changes are greater which leads to an increase in packet delivery ratio. Similarly, NRGSN and GAP are not completely identical. In the exactly-one-destination scenario, because of finding best route to nearest destination, packet delivery ratio is more than that of the other two scenarios. The lowest packet delivery ratio belongs to K-anycast destination scenario. Because the purpose is to deliver packets to K best destination from a set of destinations. Fig. 28, 30, and 32 show packet delivery ratio by a various mobility speed of nodes in three scenarios. As node mobility speed rises, packet delivery ratio falls. This reduction is expected considering fast changes of topology and routes in each step. The change process of diagrams GAP and NRGSN is similar. According to resembling explanations, the trend of the diagrams related to the proposed algorithm and GKAR in [13], are similar.

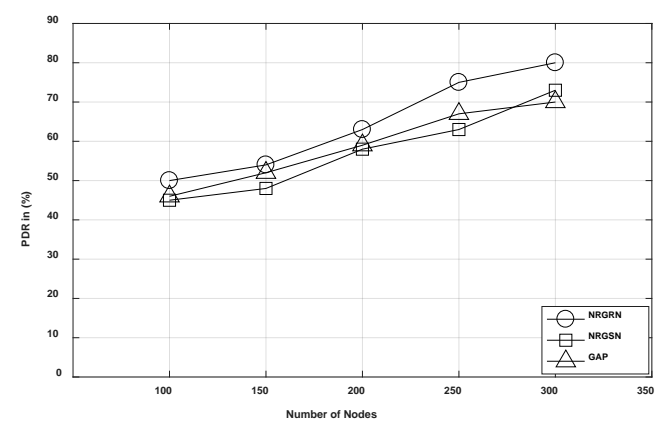

Fig. 27. Packet Delivery Ratio with various numbers of nodes (exactly-one-destination scenario)

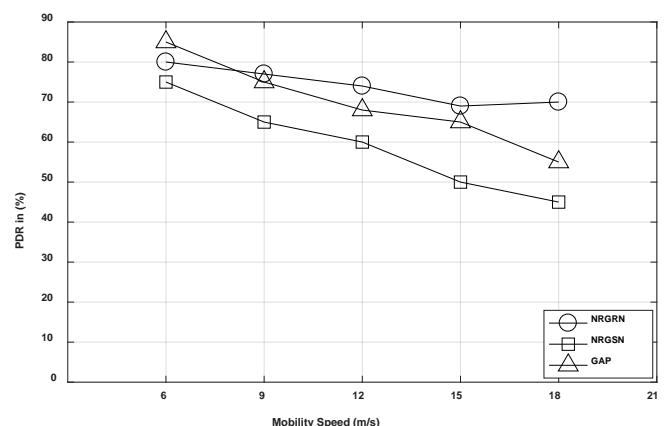

Fig. 28. Packet Delivery Ratio with various mobility speed (exactly-one-destination scenario) 


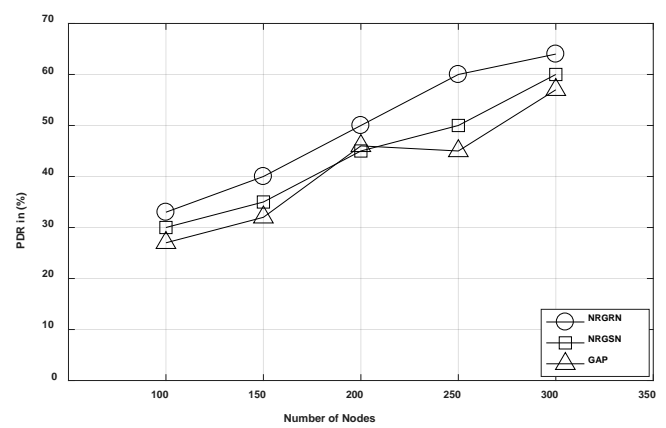

Fig. 29. Packet Delivery Ratio with different numbers of nodes (at-least-one-destination scenario)

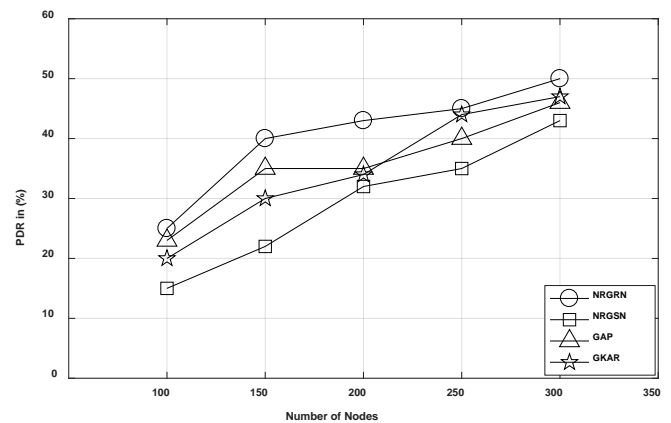

Fig. 31. Packet Delivery Ratio with different numbers of nodes (K-anycast destination scenario)

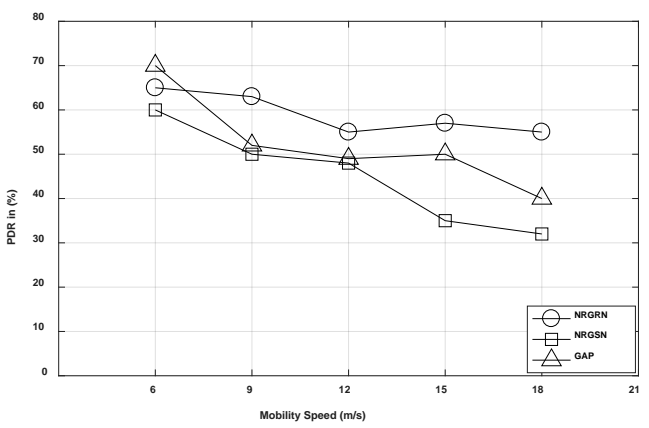

Fig. 30. Packet Delivery Ratio with different numbers of nodes (at-least-one-destination scenario)

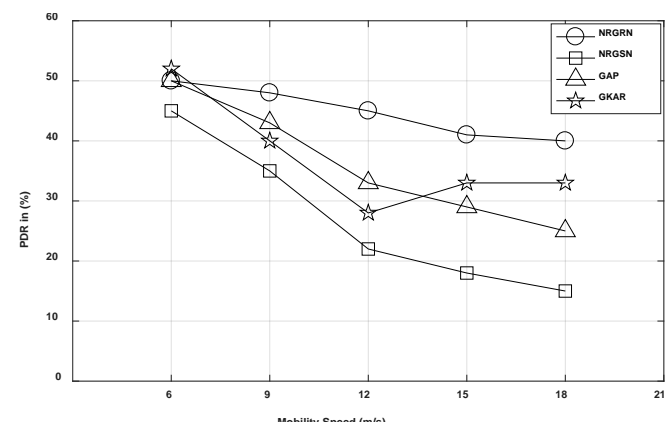

Fig. 32. Packet Delivery Ratio with different mobility speed (K-anycast destination scenario)

\section{Total evaluation}

In order to have a total evaluation, metrics including packet delivery ratio, the average hop count, the average delivery delay, and the average delivery cost were investigated and compared under the condition of 150 nodes and average speed of $9(\mathrm{~m} / \mathrm{s})$ for each of the scenarios. Fig. 33 shows packet delivery ratio in three scenarios. The excactly one destination scenario includes the high packet delivery ratio since the best destination is selected from the beginning, and the packet is delivered to the same destination. In at-least-one-destination scenario, as there is more than one destination, each node may have to produce and forward one or more packet that leads to an increase in packet delivery ratio; packet delivery ratio will be lower than the first scenario. In K-anycast destination scenario, in which the purpose is to send packet to K-number of best destinations from among a set of destinations, packet delivery is more demanding and packet delivery ratio will be lower than that of the two other scenarios.

Fig. 34 shows the average hop count. The average hop count in the first scenario will be the least because there is one defined destination. In at-least-one-destination scenario, similarly, the average number of hops is more than that in the first scenario. In K-anycast destination scenario, we have the highest number of hops because there are K-destinations. 


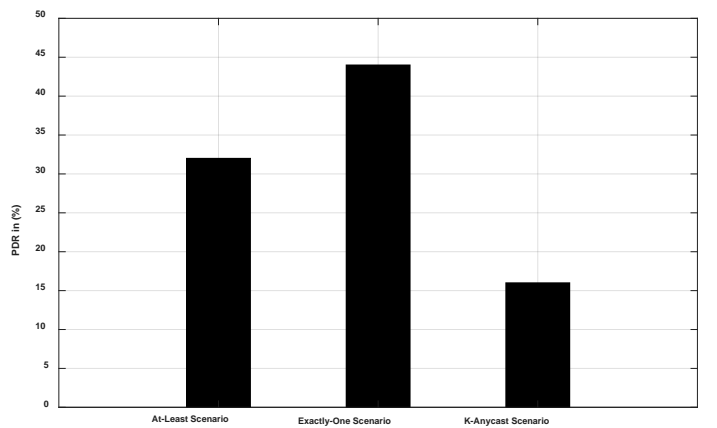

Fig. 33. Packet Delivery Ratio in different scenarios

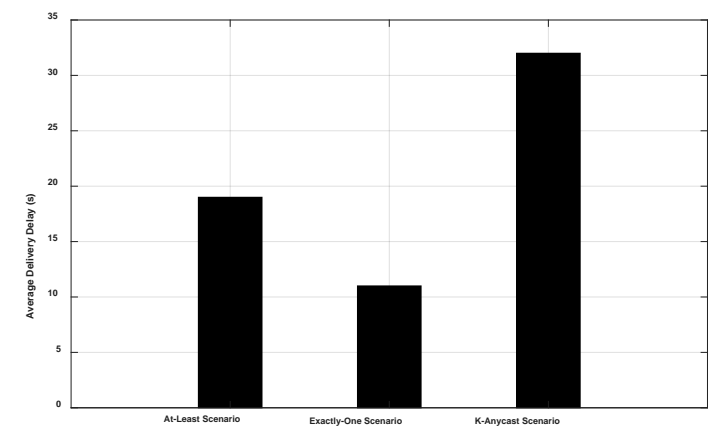

Fig. 35. Average Packet Delivery Delay in different scenarios.

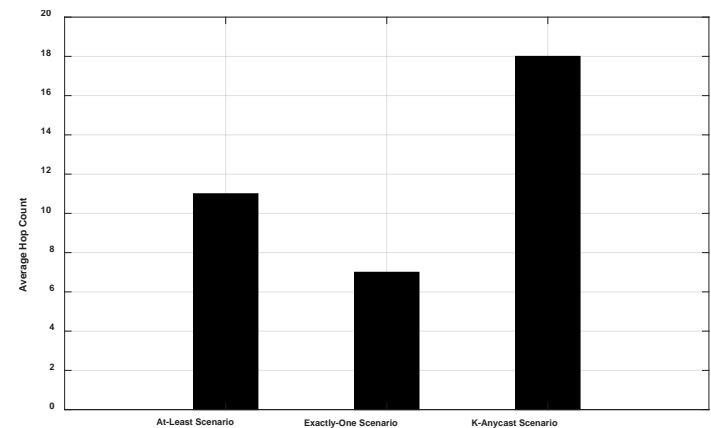

Fig. 34. Average Hop Count in different scenarios.

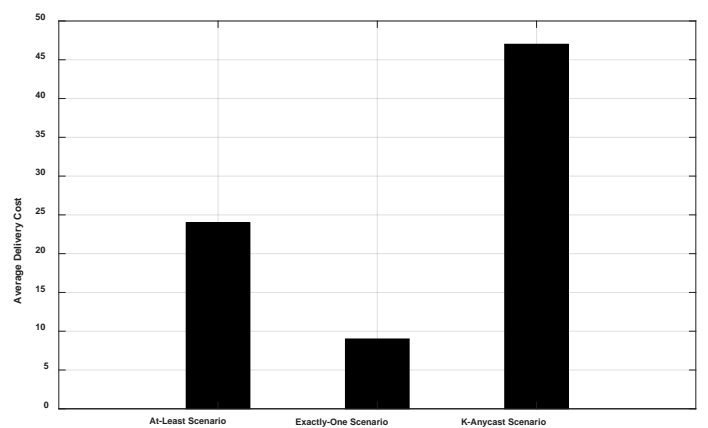

Fig. 36. Average Packet Delivery Cost in different scenarios.

Fig. 35 shows the average packet delivery delay. Exactly one destination scenario has the lowest delay because, from the start, the destination is defined (delay is related to the packet arriving at the destination). In at-least-one-destination, the average packet delivery delay will be higher than that in the first scenario (the probability of producing more than one packet by a defined node because destinations are more than one). In K-anycast destination scenario, the average packet delivery delay is higher than that in the two other scenarios because the process comes to an end when the packets are delivered to $\mathrm{K}$ destinations.

Fig. 36 shows the average packet delivery cost. In exactly-one-destination scenario, considering that destination is defined, the route with fewer hops is selected and the average packet delivery cost will be lower than that in the other two scenarios. At-least-one-destination scenario has higher average packet delivery cost compared to the exactly one destination scenarios because some nodes have to forward more extra packets and as result number of hops increase. K-anycast destination scenario has the higher number of hops and, the higher the average packet delivery cost compared to the other two scenarios. Because packets must be delivered to $\mathrm{K}$ destination.

\section{Conclusion}

Dynamic topology of vehicular networks has originated from vehicle mobility. That is why we cannot model these networks as graphs. Therefore, mathematical modeling is hardly possible. In this article, applying time-expanded networks, we could model a vehicular network.

Anycast communication pattern can cover various applications of vehicular networks. As geographical routing protocols in vehicular networks are more efficient and popular, this article focused on geographic anycast. Three different scenarios were presented for anycast and any of these scenarios can cover a bunch of vehicular networks applications. 
(Transmitting anycast packets to exactly one destination, to at-least-one-destination, and to $\mathrm{K}$-anycast destination). Based on the presented modeling for vehicular networks, geographic anycast in vehicular networks was modeled by applying mixed-integer linear programming. For each scenario, a single mathematical model was proposed and based on the model, different metrics were presented to evaluate it. The evaluation metrics include packet delivery ratio, average packet delivery delay, average packet delivery cost, and average hop count.

Proposed models are of MILP type and are in NP domain and need a decentralized algorithm to be applied in vehicular networks; a heuristic algorithm was presented that selected the best neighbor(s) to forward geographic anycast packets to the destination(s).

The evaluation process of this article includes the numerical evaluation of the proposed model by Branch and Bound algorithm by GAMS software and also simulation of the proposed algorithm by OMNET++ simulator.

The proposed metrics were investigated for these three scenarios. The average packet delivery ratio related to exactly-one-destination scenario was higher than that of the other scenarios. The average hop count, the average packets delivery delay, and the average packet delivery cost in K-anycast destination scenario was higher than those in the other scenarios.

It was observed that there were similar changes in evaluation metrics in the results obtained from simulator and the results of realistic model obtained from GAMS. In other words, the conformity of proposed algorithm and presented model refers to the acceptable accuracy of the project.

Considering the similar behavior in the proposed algorithm and geographic K-anycast destination routing in [13], similar variations in their diagrams were observed.

This study, done on modeling vehicular networks by time-expanded networks, can create a framework to present future protocols for these networks. It may be helpful to overcome the main challenge of vehicular or simply its highly dynamic topology.

For further studies, considering new communication technologies specially IoV, the proposed models and protocol can be adjusted and applied to IoV. For instance, anycast communication patterns can be applied as a gateway selection in IoV.

\section{References}

[1] Urquiza-Aguiar. L, Tripp-Barba. C, Aguilar Igartua. M, “A geographical heuristic routing protocol for VANETs,” Sensors, Vol. 16, no 10, pp.1567, 2016. Article (CrossRef Link)

[2] Wu. C, Yoshinaga. T, Bayar. D, Ji. Y, "Learning for adaptive anycast in vehicular delay tolerant networks," Journal of Ambient Intelligence and Humanized Computing, Vol. 10, no 4, pp.1379-1388, 2019. Article (CrossRef Link)

[3] Wu. Q, Liu. Q, Zhang. L, Zhang. Z, "A trusted routing protocol based on GeoDTN+ Nav in VANET,"China Communications, Vol. 11, no 14, pp. 166-174, 2014. Article (CrossRef Link)

[4] Li. W, Chenyu. G, Gu. D, Liao. L, Gao. Z, Shi. Z, Lu. T, Liu. Y, Liu. Z "Security Analysis of the Khudra Lightweight Cryptosystem in the Vehicular Ad-hoc Networks," KSII Transactions on Internet and Information Systems (TIIS), Vol. 12, no 7, pp. 3421-3437, 2018.

Article (CrossRef Link)

[5] Kaur. H, "Analysis of VANET geographic routing protocols on real city map,” in Proc. of 2017 2nd IEEE International Conference on Recent Trends in Electronics, Information \& Communication Technology (RTEICT), pp. 895-899, 2017. Article (CrossRef Link)

[6] Al-Mashaqbeh. GA, Al-Karaki. JN, Al-Rousan. M, Raza. A, Abbas. H, Pasha. M., "Joint Geographic and Energy-aware Routing Protocol for Static and Mobile Wireless Sensor Networks," Adhoc \& Sensor Wireless Networks, vol. 41, no. 3/4, pp. 165-190, 2018. Article (CrossRef Link)

[7] Oubbati. O.S, Lakas. A, Zhou. F, Güneş. M, Lagraa. N, Yagoubi. M.B, "Intelligent UAV-assisted routing protocol for urban VANETs,” Computer communications, vol. 107, pp. 93-111, 2017. Article (CrossRef Link) 
[8] Zhang. L, Cao. W, Zhang. X, Xu. H, "MAC²: Enabling multicasting and congestion control with multichannel transmission for intelligent vehicle terminal in Internet of Vehicles,” International Journal of Distributed Sensor Networks, vol. 14, no. 8, 2018. Article (CrossRef Link)

[9] Cheng. J, Cheng. J, Zhou. M, Liu. F, Gao. S, Liu. C, "Routing in internet of vehicles: A review," IEEE Transactions on Intelligent Transportation Systems, Vol. 16, no. 5, pp. 2339-2352, 2015. Article (CrossRef Link)

[10] Ren. C, Wang. S, Ren. J, Wang. X, “Traffic Engineering and Manageability for Multicast Traffic in Hybrid SDN," KSII Transactions on Internet \& Information Systems, Vol. 12, no 6, pp. 2492-2512, 2018. Article (CrossRef Link)

[11] Le. T, Gerla. M, “Time-constrained anycast routing under short contact duration in delay-tolerant networks,” Annals of Telecommunications, Vol. 73, no. 9-10, pp. 549-558, 2018. Article (CrossRef Link)

[12] Oak. J. Y, Choi. Y. J., Pak. W, "EP-MAC: Early Preamble MAC To Achieve Low Delay And Energy Consumption In Duty Cycle Based Asynchronous Wireless Sensor Networks,” KSII Transactions on Internet \& Information Systems, Vol. 6, no. 11, pp. 2980-2991, 2012. Article (CrossRef Link)

[13] Wang. X, Wang. J, Lu. K, Xu. Y, “GKAR: A novel geographic K-anycast destination routing for wireless sensor networks,” IEEE Transactions on Parallel and Distributed Systems, Vol. 24, no. 5, pp. 916-925, 2013. Article (CrossRef Link)

[14] Kumari. N.D, Shylaja. B.S, "AMGRP: AHP-based multimetric geographical routing protocol for urban environment of VANETs," Journal of King Saud University-Computer and Information Sciences, vol. 31, no. 1, pp. 72-81, 2019. Article (CrossRef Link)

[15] Sharma S, Kaushik. B, “A survey on internet of vehicles: Applications, security issues \& solutions,” Vehicular Communications, Vol. 20, 2019. Article (CrossRef Link)

[16] Qureshi. KN, Abdullah. AH, Lloret. J, "Road perception based geographical routing protocol for vehicular ad hoc networks,” International Journal of Distributed Sensor Networks, Vol. 12, no. 2, 2016. Article (CrossRef Link)

[17] Ghafoor. KZ, Lloret. J, Sadiq. AS, Mohammed. MA, "Improved geographical routing in vehicular ad hoc networks,” Wireless Personal Communications, Vol. 80, no. 2, pp. 785-804, 2015. Article (CrossRef Link)

[18] Ghafoor. KZ, Abu Bakar. K, Lloret. J, Khokhar. RH, Lee. KC, "Intelligent beaconless geographical forwarding for urban vehicular environments,” Wireless networks, Vol. 19, no. 3, pp. 345-62, 2013. Article (CrossRef Link)

[19] Cao. Y, Wang. T, Zhang. X, Kaiwartya. O, Eiza. MH, Putrus. G, “Toward anycasting-driven reservation system for electric vehicle battery switch service,” IEEE Systems Journal, Vol. 13, no. 1, pp. 906-917, 2019. Article (CrossRef Link)

[20] Le. T, Gerla. M. "Social-distance based anycast routing in delay tolerant networks," in Proc. of 2016 Mediterranean Ad Hoc Networking Workshop (Med-Hoc-Net), pp. 1-7, 2016. Article (CrossRef Link)

[21] Baguena. M, Miguel, Calafate. C.T, Cano. J.C, Manzoni. P, “An adaptive anycasting solution for crowd sensing in vehicular environments,” IEEE Transactions on Industrial Electronics, Vol. 62, no 12, pp. 7911-7919, 2015. Article (CrossRef Link)

[22] Gao. D, Lin. H, Liu. X, "Routing Protocol for K-anycast Communication in Rechargeable Wireless Sensor Networks,” Computer Standards \& Interfaces, Vol. 43, pp.12-20, 2016. Article (CrossRef Link)

[23] Dixit. RK, Johari. R, "As-puma: anycast semantics in parking using metaheuristic approach,” International Journal on AdHoc Networking Systems (IJANS), Vol. 3, no. 3, July 2013. Article (CrossRef Link)

[24] Urquiza-Aguiar. L, Igartua. MA, Tripp-Barba. C, Calderón-Hinojosa. X, "2hGAR: 2-hops geographical anycast routing protocol for vehicle-to-infrastructure communications," in Proc. of the 15th ACM International Symposium on Mobility Management and Wireless Access, pp. 145-152, 2017. Article (CrossRef Link)

[25] Le. T, Gerla. M, “An anycast routing strategy with time constraint in delay tolerant networks,” in Proc. of 2017 16th Annual Mediterranean Ad Hoc Networking Workshop (Med-Hoc-Net), pp. 1-6, 2017. Article (CrossRef Link) 
[26] Li. Y, Wang. X, Dou. Z, “k-Anycast data acquisition in multi-hop IoV,” Journal of High Speed Networks, Vol. 25, no. 2, pp. 173-180, 2019. Article (CrossRef Link)

[27] Nelson. SC, Hu. YC, Kravets. R, “Anycast, multicast and beyond: the role of manycast in DTN communication,” IDEALS, pp. 1-13, 2011. Article (CrossRef Link)

[28] Amirshahi. A, Romoozi. M, Raayatpanah. M.A, Asghari. S.A, "Anycast routing in time-expanded vehicular networks," Computers \& Electrical Engineering, Vol. 82, 2020. Article (CrossRef Link)

[29] Skutella. M., “An introduction to network flows over time,” Research trends in combinatorial optimization, Springer, pp. 451-482, 2009. Article (CrossRef Link)

[30] Ghasvari. H, Raayatpanah. M.A, Khalaj. B.H, Bakhshi. H, "Optimal sub-graph selection over coded networks with delay and limited-size buffering,” IET communications, Vol. 5, no 11, pp. 1497-1505, 2011. Article (CrossRef Link)

[31] Bisengar. A, Zytoune. O, Rziza. M, Ouadou. M, Aboutajdine. D, “A Routing Protocol Based on Mobility Prediction for Mobile Ad Hoc Networks,” Journal of Communications Software and Systems, Vol. 10, no. 1, pp. 24-29, 2014. Article (CrossRef Link)

[32] Romoozi. M, Fathy. M, Berangi. R, "Performance analysis and improvement content discovery protocols over vehicular networks," Wireless personal communications, Vol. 75, no. 2, pp. 857-899, 2014. Article (CrossRef Link)

[33] Zhang. K, Kim. J, Cho. G, “An Efficient and Energy-saving Data Dissemination Mechanism for Low-power and Lossy Networks," IEIE Transactions on Smart Processing \& Computing, Vol. 7, no. 4, pp. 271-278, 2018. Article (CrossRef Link) 


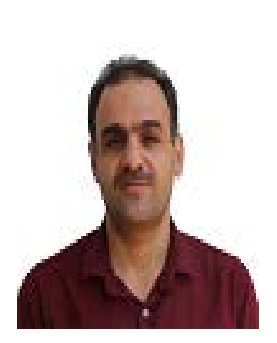

Alireza Amirshahi received the B.S. degree in Computer Engineering from Kashan Azad University, Kashan, Iran 2002, the M.S. degree in computer Architecture from Arak Azad University, Arak, Iran, in 2011. He is a PhD student in computer Architecture at Qom Azad University, Iran, Qom, in 2014. His research interests include the Vehicular Network, Intelligent Transportation Systems, Wireless Ad-hoc Networks, and Wireless Sensor Network.

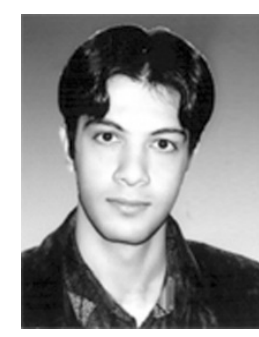

Morteza Romoozi received the B.S. degree in Software Engineering from Kashan University, Kashan, Iran, in 2003, the M.S. degree in software engineering from Arak Open University, Arak,Iran, in 2008, and the Ph.D. degree in computer systems from the Science and Research Branch of Azad University, Tehran, Iran, in 2013. He is assistant professor at Department of Computer Engineering, Azad University, Kashan Branch. His research interests include the Vehicular Network, Intelligent Transportation Systems, P2P Networks, Wireless Ad-hoc Networks, Wireless Sensor Network, Cloud Computing and Semantic Information Retrieval.

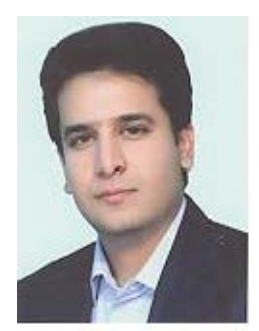

Mohammad Ali Raayatpanah received his B.Sc. degree from Kashan University, Esfahan, Iran, in 2004, and the M.Sc. degree from Tehran University, Tehran, Iran, in 2006, in Applied Mathematics. He also received his $\mathrm{PhD}$ degree in Applied Mathematics from the department of mathematics, statistics and computer science of the University of Tehran, in 2013. He is currently an assistant professor in the Department of Mathematical and Computer Sciences, Kharazmi University, Tehran, Iran. His current research focuses on linear programming applications on wired and wireless networks.

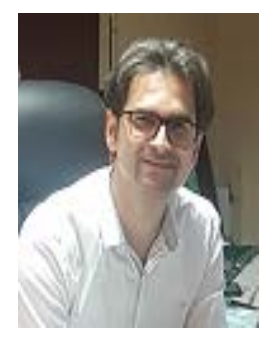

Seyyed Amir Asghari was born in Lashte Nesha, Gilan, Iran, on June 26, 1984. He received his B.S., M.S., and Ph.D. degrees in Computer Engineering from Amirkabir University of Technology, Tehran, Iran, in 2007, 2009, and 2013 respectively. His interests include reliable and fault tolerant embedded system design, real-time system design, and operating systems. He is currently the assistant professor of faculty of engineering in Kharazmi University of Tehran. 